\title{
Alternative method to predict activity coefficients at infinite dilution of hydrocarbons in aqueous solutions
}

\author{
Gonzalo Astray $^{1,2, *}$, Jorge Morales ${ }^{1}$, Miriam González-Temes ${ }^{1}$, Juan C. Mejuto ${ }^{1}$ \\ and Augusto J. Magdalena ${ }^{1}$ \\ ${ }^{1}$ Colloidal Chemistry Laboratory, Physical Chemistry Department, University of Vigo, As Lagoas, \\ 32004, Ourense, Spain \\ ${ }^{2}$ Department of Geological Sciences, College of Arts and Sciences, Ohio University, Clippinger Laboratories, \\ Athens, Ohio, USA
}

\begin{abstract}
Activity coefficients at infinite dilution are important property in solute-solvent interactions. Experimental techniques show high costs, skilled labour and safety. To solve this, a neural network model with five different topological descriptors to implement different Artificial Neural Network has been implemented.

The best Artificial Neural Network (5-11-8-1 topology) presents good fits for the training phase with an Average Percentage Deviation of $1.85 \%$. Similar results have been obtained for the validation phase of the Artificial Neural Network (1.88\%). The implemented Neural Networks techniques showed better results than other developed methods, around $30.70 \%$ and $24.60 \%$ for training and validation phase, respectively.
\end{abstract}

Keywords: Artificial Neural Network; infinite dilution activity coefficients; hydrocarbons.

\section{Introduction}

Activity coefficients at infinite dilution $\left(\gamma^{\infty}\right)$ are important to supply many aspects about the solute-solvent interactions ${ }^{1-3}$, the type and the strength of interactions between the solvent and the solute ${ }^{1,4}$ or the development of thermodynamic models ${ }^{2}$. They can also provide us information about the design of separation and extraction processes ${ }^{2,5}$ or the selection of solvents for rectification and extraction ${ }^{2}$, the behaviour of ionic liquids ${ }^{6}$, the prediction for azeotrope formations ${ }^{7}$, the mutual-solubility estimation ${ }^{8}$, etc.

There are different experimental methods to determinate the activity coefficients at infinite dilution $^{1,9,10}$ such as gas-liquid chromatography $(\mathrm{GLC})^{11}$, dilutor method ${ }^{12}$ or inverse gas chromatography $^{13}$. All these experimental techniques show some important limitations as high costs, skilled labour and safety ${ }^{1,4}$. Therefore, it would be advisable to develop predictive models that are able to determine the value of activity coefficients at infinite dilution. Nowadays, there are many theoretical models to determine this parameter ${ }^{1}$ as analytical solution of groups (ASOG) ${ }^{14-16}$ and unified functional activity coefficient UNIFAC ${ }^{17,18}$. Over the past decades, models based in quantitative structure-property relationships (QSPRs) have been proposed to estimate activity coefficients, both by multiple linear regression (MLR) and Artificial Neural Networks (ANN) ${ }^{19-24}$. QSPR models are based on the relationship between the molecular chemical descriptors and a desired physicochemical property ${ }^{19}$, in this case the activity coefficients at infinite dilution. In many cases, this method is hardly complicated due the necessity to choose between all molecular chemical descriptors that will be used to feed the prediction model. In this sense, it is necessary a method to optimize the choice of

*Corresponding author: Gonzalo Astray

E-mail address: gastray@uvigo.es

DOI: http://dx.doi.org/10.13171/mjc.3.6.2015.01.02.11.25.astray 
variables used later for the implementation of the prediction model. A good method, for the selection of the input variables, is the Ant Colony Optimization method (ACO). In fact, this method has been used in the last years to develop predictive models ${ }^{25,26}$.

In the present manuscript, a prediction model to determinate the infinite dilution activity coefficients of hydrocarbons in aqueous solutions has been developed using Artificial Neural Network based in variables selected by the Atabati's ACO method ${ }^{1}$. Artificial Neural Networks were developed for human reasoning modelling, built around an artificial neuron that simulates the biological neuron to process information ${ }^{27}$. Recently, Neural Networks have been shown as a useful tool for the prediction in various fields to solve complex problems ${ }^{27,28}$. For example, this technology can be applied in areas as medicine ${ }^{29,30}$, chemistry ${ }^{31-33}$, atmospheric sciences $^{34,35}$, or food technology ${ }^{36,37}$, inter alia.

\section{Experimental Section}

\section{Data Set}

The experimental values of infinite dilution activity coefficients at infinite dilution coefficients in water, at temperature $298.15 \mathrm{~K}$, of 105 hydrocarbons cases have been taken from literature ${ }^{1,20}$. Data set have been divided into two groups, the first one consisting in 75 cases for the Artificial Neural Networks training, and the second one of 30 cases to validate the good accuracy of the implemented Neural Networks. The topological descriptors and the two groups are exactly the same chosen by Atabati et al. ${ }^{1}$. Firstly, they used HyperChem Software to draw structures of the molecules, and secondly they calculated the different quantum-chemical descriptors using Dragon Software ${ }^{1}$. As result, five different topological descriptors have been chosen: i) IC0 corresponds with information content index (neighbourhood symmetry of 0-order); ii) ADDD corresponds with the average distance/distance degree iii) Mor10m corresponds with the 3D-Morse - signal 10/weighted by atomic masses; iv) HTm corresponds with the $\mathrm{H}$ total index/weighted by atomic masses, and finally, v) refractivity, corresponds with the relationship between the angle of incidence and refraction in a medium ${ }^{1}$.

\section{Artificial Neural Network}

A Multilayer Neural Network with an input layer to collect data of training cases has been used. The equations that govern the ANN learning are the propagation function, that transmits information between the different neurons in the different layers (Eq. 1), and the activation function, that provides an new value in different neurons (Eq. 2). $\mathrm{N}$ represents the neurons in input layer, $\mathrm{w}_{f d}$ is the weight between the $f$ neuron of input layer and the $d$ neuron in the intermediate layer, present in intermediate layer, and $b_{f}$ is the value of the "bias" neuron associated to each the intermediate neuron $f$. Then, the value which has been obtained by the propagation function was used by the activation function to provide an output value ${ }^{27,31}$.

$$
\begin{aligned}
& S_{f}=\sum_{d=1}^{N} w_{f d} x_{d}+b_{f} \\
& F_{f}\left(S_{f}\right)=\frac{1}{1+e^{-S_{f}}}
\end{aligned}
$$

The output-neuron error has been previously established by the technician. Thus, if it did not adjust to the previously established error, the process continued until the desired value. The error was used to modify the weights between different neurons. In fact, this error has 
been calculated according the Eq. 3, where $\gamma_{g}^{\infty}$ corresponds with the experimental value and $y_{g}$ is the predicted value calculated in the output neuron $g^{27,28,31}$.

$$
E=\frac{1}{2} \sum_{g=1}^{M}\left(\gamma_{g}^{\infty}-y_{g}\right)^{2}
$$

The notation used to represent and identify all neural topologies developed is: $N_{\text {input }}-N_{i 1-}$ $N_{i 2}-N_{i 3}-N_{\text {output }}$ being $N_{\text {input }}$ and $N_{\text {output }}$ the neurons present in the input and output layer and $N_{i 1}, N_{i 2}$ and $N_{i 3}$ correspond with neurons in the first, second and third intermediate layer developed in the ANN model, respectively ${ }^{27,28}$.

To choose the best Neural Network model we have used the Root Mean Square Error (RMSE) comparing predicted values $\left(y_{g}\right)$ and real values $\left(d_{g}\right)$, according to Eq. 4.

$$
R M S E=\sqrt{\sum_{i=1}^{N} \frac{\left(y_{g}-\gamma_{g}^{\alpha}\right)}{N}}
$$

Furthermore, the Average Percentage Deviations (APD) (Eq. 5) and the Individual Percentage Deviations (IPD) (Eq. 6) have been calculated in order to compare the errors of developed models.

$$
\begin{gathered}
A P D=\frac{\left(\sum_{i=1}^{N}\left(\frac{\left(y_{g}-\gamma_{g}^{\alpha}\right)}{\gamma_{g}^{\alpha}}\right)\right) \cdot 100}{N} \\
I P D=\frac{\left(y_{g}-\gamma_{g}^{\alpha}\right)}{\gamma_{g}^{\alpha}} \cdot 100
\end{gathered}
$$

\section{Results and Discussion}

\section{Training phase of ANN}

The Artificial Neural Networks activity requires a high implementation of networks using the trial and error method to obtain the best Neural Network to determine more accurately the activity coefficients at infinite dilution. Due this, five hundred Neural Networks has been implemented, changing its topology and its training cycle numbers.

Table 1 shows the top 5 Neural Networks implemented. This table shows the linear fit coefficient $\left(\mathrm{R}_{\mathrm{T}}^{2}\right)$, the Root Mean Square Error $\left(\mathrm{RMSE}_{\mathrm{T}}\right)$, and Average Percentage Deviation $\left(\mathrm{APD}_{\mathrm{T}}\right)$ in the training phase. Table 1 shows a neural network with a lower RMSE and it presents a 5-11-10-1 topology. This Neural Network presents a $\mathrm{R}^{2}$ coefficient of 1.000 with a $\mathrm{RMSE}_{\mathrm{T}}=0.018$ that represents a $0.085 \%$ Average Percentage Deviation for the training phase. The best Neural Network consists in a Neural Network with five neurons in the input layer, two intermediate layers with eleven and ten neurons respectively, and finally, an output layer with only a neuron corresponding to the variable that we try to predict (the value of activity coefficients at infinite dilution). 
Table. 1. Fits of the five best ANN implemented for the training phase using different ANN architectures. $\mathrm{R}^{2}$ represents the square correlation coefficient, $\mathrm{RMSE}_{\mathrm{T}}$, the Root Mean Square Error and $\mathrm{APD}_{\mathrm{T}}$, the Average Percentage Deviation.

\begin{tabular}{ccccc}
\hline Cycles & Topology & $\mathbf{R}_{\mathbf{T}}^{\mathbf{2}}$ & $\mathbf{R M S E}_{\mathbf{T}}$ & $\mathbf{A P D}_{\mathbf{T}}$ \\
\hline $1,024,000$ & $\mathbf{5 - 1 1 - 1 0 - 1}$ & 1.000 & 0.018 & 0.085 \\
$1,024,000$ & $\mathbf{5 - 1 1 - 7 - 1}$ & 1.000 & 0.019 & 0.098 \\
$1,024,000$ & $\mathbf{5 - 1 1 - 8 - 1}$ & 0.999 & 0.030 & 0.148 \\
512,000 & $\mathbf{5 - 1 1 - 9 - 1}$ & 0.999 & 0.031 & 0.113 \\
$1,024,000$ & $\mathbf{5 - 7 - 6 - 1}$ & 0.999 & 0.034 & 0.226 \\
\hline
\end{tabular}

\section{Validation phase of ANN}

Once the adjustment of different ANN for the training phase were calculated, we have proceeded to fit the validation phase and we ordered the ANN from lowest to highest validation RMSE $\left(\mathrm{RMSE}_{\mathrm{V}}\right)$. In this case, 5-11-8-1-topology was the best Neural Network with a lower RMSE (0.291). Others fits of this ANN presented good results with a $\mathrm{R}^{2}=0.986$ and a $1.875 \%$ of APD (Table 2). This network had five neurons in the input layer, two intermediate layers (the first with eleven neurons and a second intermediate layer eight neurons), and finally, an output layer with a single neuron.

Table. 2. Fits of the five best ANN implemented for the validation phase using different ANN architectures. $\mathrm{R}_{\mathrm{V}}^{2}$ represents the square correlation coefficient, $\mathrm{RMSE}_{\mathrm{V}}$, the Root Mean Square Error and $\mathrm{APD}_{\mathrm{V}}$, the Average Percentage Deviation.

\begin{tabular}{ccccc}
\hline Cycles & Topology & $\mathbf{R}_{\mathbf{V}}^{2}$ & $\mathbf{R M S E}_{\mathbf{V}}$ & $\mathbf{A P D}_{\mathbf{V}}$ \\
\hline 64,000 & $\mathbf{5 - 1 1 - 8 - 1}$ & 0.986 & 0.291 & 1.875 \\
32,000 & $\mathbf{5 - 7 - 1}$ & 0.983 & 0.307 & 2.084 \\
16,000 & $\mathbf{5 - 1 1 - 7 - 1}$ & 0.984 & 0.316 & 1.811 \\
16,000 & $\mathbf{5 - 7 - 4 - 1}$ & 0.984 & 0.317 & 1.944 \\
64,000 & $\mathbf{5 - 7 - 1}$ & 0.982 & 0.321 & 2.310 \\
\hline
\end{tabular}

\section{Best Artificial Neural Network implemented}

The aim of the present manuscript was to implement a tool based on Neural Networks that would be able to predict the activity coefficients values at infinite dilution of various hydrocarbons. If we choose the Neural Network that has the lowest RMSE in the training phase, the network only fits perfectly with the cases that have been trained but not with other patterns that it had not been previously trained. In order to predict the activity coefficients at infinite dilution to untrained cases, we had to choose a Network with the lowest RMSE in the validation phase. Therefore, we chose a network with a 5-11-8-1 topology (Table 3).

From Fig. 1 we can see the experimental activity coefficients at infinite dilution versus the predicted values our neural network model. As it can be observed, these results are satisfactory according the line of slope one. Moreover we can show the Average Percentage Deviation for training phase at Fig. 2. The Neural Network chosen (5-11-8-1) has an $\mathrm{APD}=1.84 \%$, with a minimum error of $-8.18 \%$ and a maximum error of $6.59 \%$ for the training phase (Fig. 2). In comparison with the MLR model that represents an APD of $2.70 \%$, with a minimum error of $-15.75 \%$ and a maximum error of $9.32 \%$. 
Table. 3. Topology of the five best Neural Networks implemented using different ANN architectures. $R^{2}$ represents the square correlation coefficient for training $\left(\mathrm{R}_{\mathrm{T}}^{2}\right)$, and validation phase $\left(\mathrm{R}_{\mathrm{V}}^{2}\right)$, Root Mean Square Errors of training $\left(\mathrm{RMSE}_{\mathrm{T}}^{2}\right)$ and validation phase $\left(\mathrm{RMSE}_{\mathrm{V}}^{2}\right)$ and Average Percentage Deviation for training $\left(\mathrm{APD}_{\mathrm{T}}\right)$ and validation phase $\left(\mathrm{APD}_{\mathrm{V}}\right)$.

\begin{tabular}{cccccccc}
\hline Cycles & Topology & $\mathbf{R}_{\mathbf{T}}^{\mathbf{2}}$ & $\mathbf{R M S E}_{\mathbf{T}}$ & $\mathbf{A P D}_{\mathbf{T}}$ & $\mathbf{R}_{\mathbf{V}}^{\mathbf{2}}$ & $\mathbf{R M S E}_{\mathbf{V}}$ & $\mathbf{A P D}_{\mathbf{V}}$ \\
\hline 64,000 & $\mathbf{5 - 1 1 - 8 - 1}$ & 0.990 & 0.277 & 1.843 & 0.986 & 0.291 & 1.875 \\
32,000 & $\mathbf{5 - 7 - 1}$ & 0.987 & 0.317 & 2.133 & 0.983 & 0.307 & 2.084 \\
16,000 & $\mathbf{5 - 1 1 - 7 - 1}$ & 0.979 & 0.397 & 2.568 & 0.984 & 0.316 & 1.811 \\
16,000 & $\mathbf{5 - 7 - 4 - 1}$ & 0.984 & 0.350 & 2.392 & 0.984 & 0.317 & 1.944 \\
64,000 & $\mathbf{5 - 7 - 1}$ & 0.989 & 0.286 & 1.888 & 0.982 & 0.321 & 2.310 \\
\hline
\end{tabular}

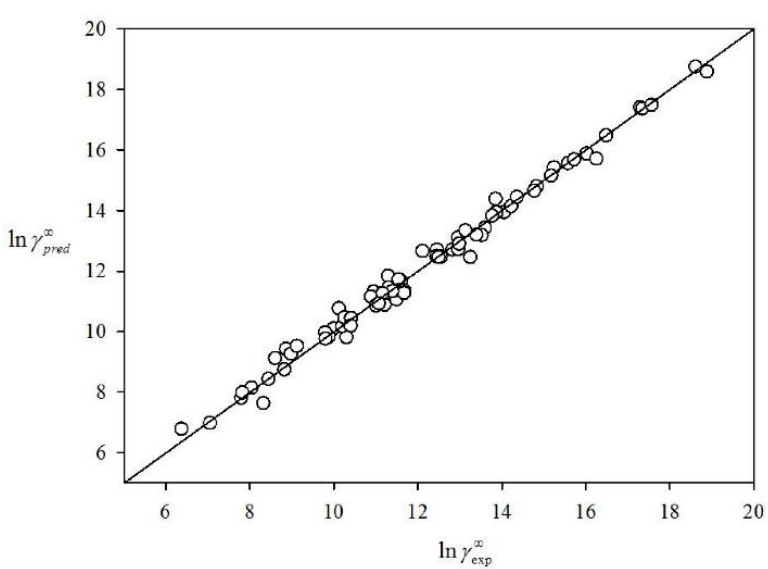

Fig. 1. Experimental Values for activity coefficients at infinite dilution $\left(\ln \gamma^{\infty} \text { exp }\right)^{1,20}$ versus predicted values $\left(\ln \gamma_{\text {pred }}^{\infty}\right)$ for the training phase of the 5-11-8-1 Neural Network. Black line represents the line of slope one

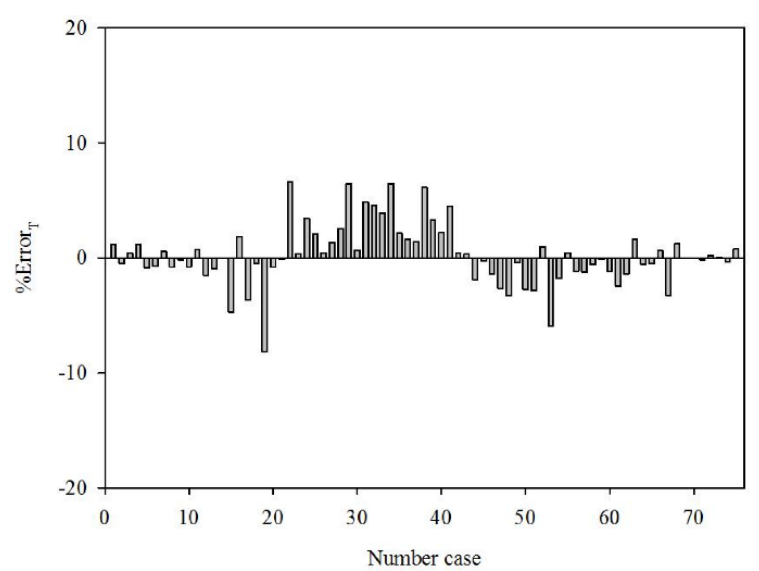

Fig. 2. Average Percentage Deviation for each cases of the ANN training phase (5-11-8-1).

The study of Atabati et al. concluded with a great model to predict this property ${ }^{1}$. Therefore, the new tool should improve the existing method. Consequently, we will analyse the main differences between our model and the model developed by Atabati et al. ${ }^{1}$. Table 4 shows the training phase fits of the two implemented models, firstly the Atabati et al. model ${ }^{1}$, and secondly the model based in ANN developed in this paper. The improvement in terms of RMSE for the training phase is very significant. In fact, it is around $30.7 \%$, representing in terms of APD about $31.4 \%$.

Table. 4. Fits for training phase, for each of the prediction models, Atabati et al. ${ }^{1}$, and Neural Network model implemented in the present article. Data used to make fits ACO-MLR model are show in Atabati et al. ${ }^{1}$.

\begin{tabular}{ccccc}
\hline Model & Topology & $\mathbf{R}_{\mathbf{T}} \mathbf{T}_{\mathbf{2}}$ & $\mathbf{R M S E}_{\mathbf{T}}$ & $\mathbf{A P D}_{\mathbf{T}}$ \\
\hline ACO-MLR $^{1}$ & & 0.979 & 0.400 & 2.689 \\
ANN & $5-11-8-1$ & 0.990 & 0.277 & 1.843 \\
\cline { 3 - 5 } Improvement & & & $30.7 \%$ & $31.4 \%$ \\
\hline
\end{tabular}

As shown, the Neural Network model presents better fits than those obtained by Atabati et al. (see Figs. 3 and 4$)^{1}$. 


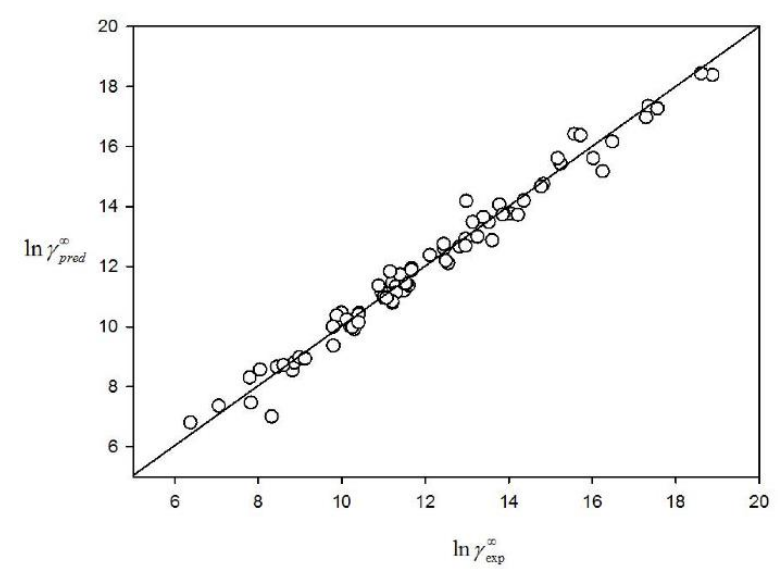

Fig. 3. Experimental values for activity coefficients at infinite dilution $\left(\ln \gamma_{\text {exp }}^{\infty}\right)^{1,20}$ versus predicted values $\left(\ln \gamma^{\infty}\right.$ pred $)$ for the training phase of ACO-MLR model ${ }^{1}$. Black line represents the line of slope one

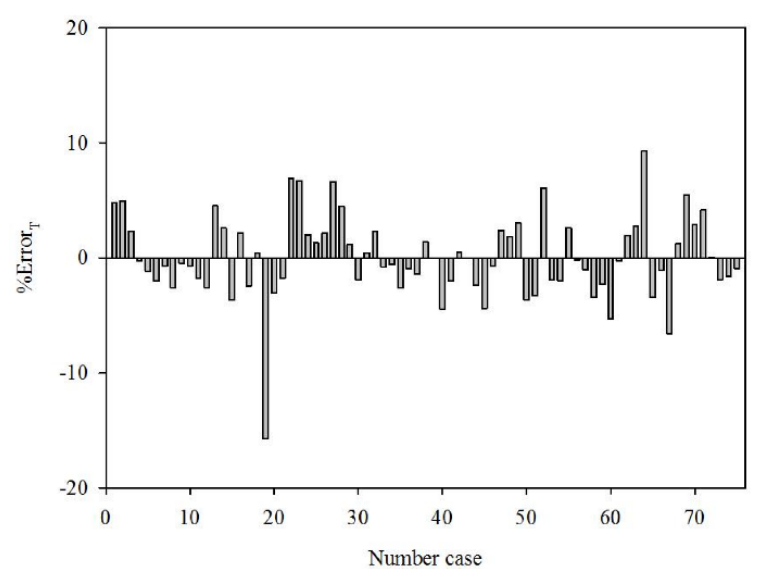

Fig. 4. Average Percentage Deviation for each cases of the ACO-MLR training phase ${ }^{1}$.

The next step is the validation of the chosen Neural Network with 30 previously reserved cases. These cases are the same used by Atabati et al. ${ }^{1}$. It is known that the Neural Network, in theory, should be the most powerful predictor for cases previously untrained. The experimental activity coefficients versus the predicted values of our model are represented in Fig. 5. All selected cases for the validation phase show good fits, not having outliers, and hence, being all successfully predicted. The individual error percentage for each validation case can be observed in Fig. 6.

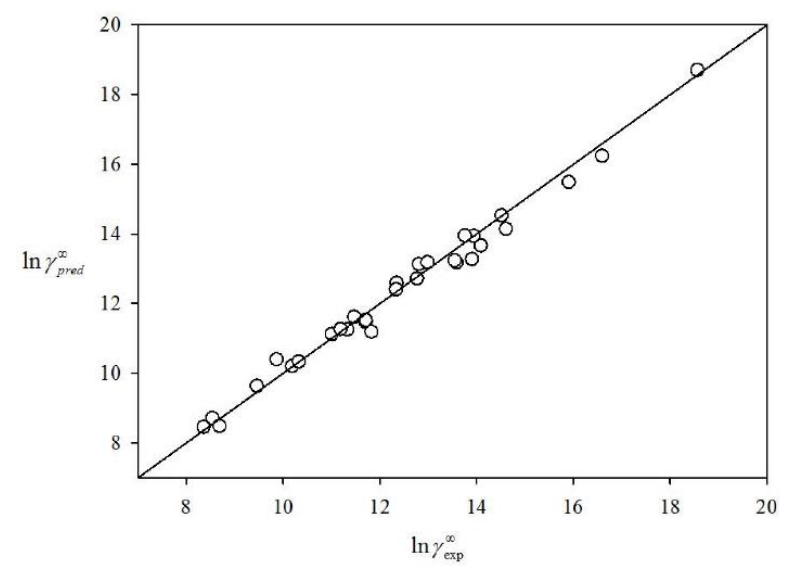

Fig. 5. Experimental values for activity coefficients at infinite dilution $\left(\ln \gamma_{\text {exp }}^{\infty}\right)^{1,20}$ versus predicted values $\left(\ln \gamma^{\infty}\right.$ pred $)$ for the validation phase of the 5-11-8-1 Neural Network. Black line represents the line of slope one.

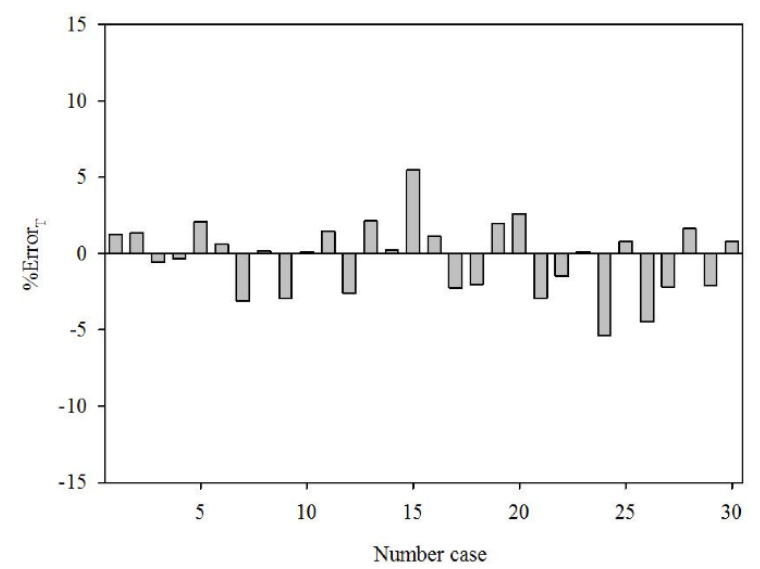

Fig. 6. Average Percentage Deviation for each cases of the ANN validation phase (5-11-8-1).

This Neural Network has an APD of $1.88 \%$, with a minimum error of $-5.35 \%$, and a maximum error of $5.45 \%$ (Fig. 6) while the MLR model has an APD of $2.64 \%$, with a minimum error of $-5.08 \%$, and a maximum error of $12.70 \%$.

Table 5 shows the validation phase fits for the models implemented, the model of Atabati et al. ${ }^{1}$ and the model based in Neural Networks, respectively. As in the previous case, the improvements for ANN are significant. It is approximately around $24.6 \%$ in terms of RMSE corresponding with $28.9 \%$ in terms of Average Percentage Deviation. 
Table. 5. Fits for validation phase, for each of the prediction models, Atabati et al. ${ }^{1}$, and Neural Network model implemented in this article. Data used to make fits ACO-MLR model are show in Atabati et al. ${ }^{1}$.

\begin{tabular}{ccccc}
\hline Model & Topology & $\mathbf{R}_{\mathbf{V}} \mathbf{V}^{\mathbf{y}}$ & $\mathbf{R M S E}_{\mathbf{V}}$ & $\mathbf{A P D}_{\mathbf{V}}$ \\
\hline ACO-MLR $^{1}$ & & 0.978 & 0.387 & 2.638 \\
ANN & $5-11-8-1$ & 0.986 & 0.291 & 1.875 \\
\cline { 3 - 5 } Improvement & & & $24.6 \%$ & $28.9 \%$ \\
\hline
\end{tabular}

Figs. 7 and 8 show the graphical fits for the model developed by Atabati et al. ${ }^{1}$. If we compare Fig. 5 (ANN model) and 7 (Atabati et al. model), in terms of trend line, the Neural Network's fits are closer than the Atabati's model ${ }^{1}$. A significant improvement of the model based on Neural Networks can be observed in some cases.

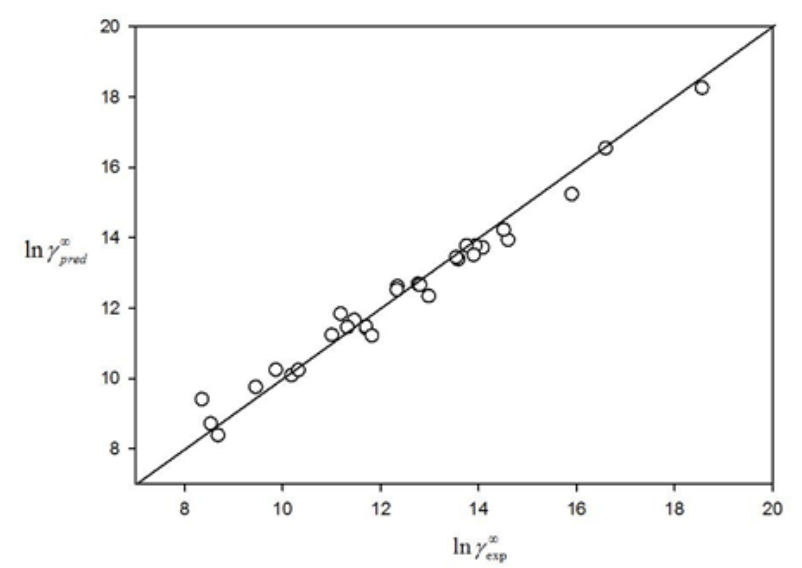

Fig. 7. Experimental values for activity coefficients at infinite dilution $\left(\ln \gamma_{\text {exp }}^{\infty}\right)^{1,20}$ versus predicted values $\left(\ln \gamma_{\text {pred }}^{\infty}\right)$ for the validation phase of ACO-MLR model ${ }^{1}$. Black line represents the line of slope one.

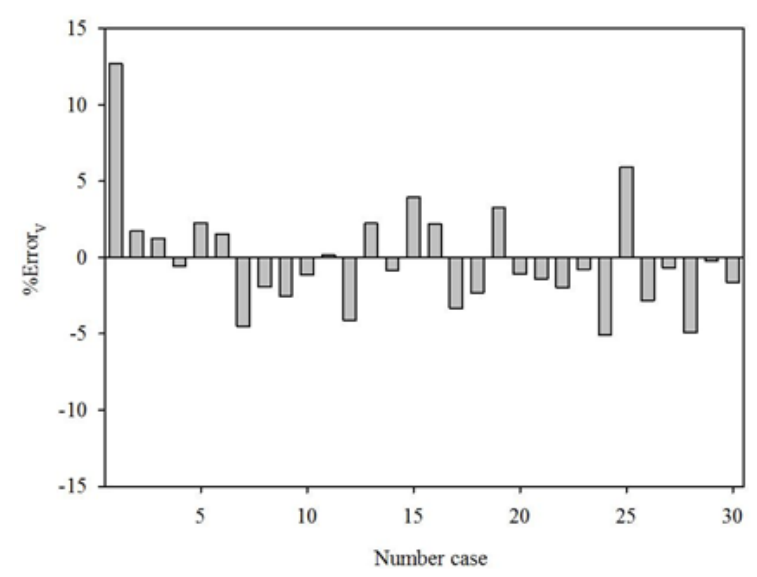

Fig. 8. Average Percentage Deviation for each cases of the ACO-MLR validation phase ${ }^{1}$.

Fig. 9 shows the selected Neural Network where it can be observed the architecture of the Neural Network with five neurons in the input layer, the nineteen neurons distributed in two intermediate layers (eleven neurons in the first layer and eight neuron in the second layer) and the output layer consisting of one neuron.

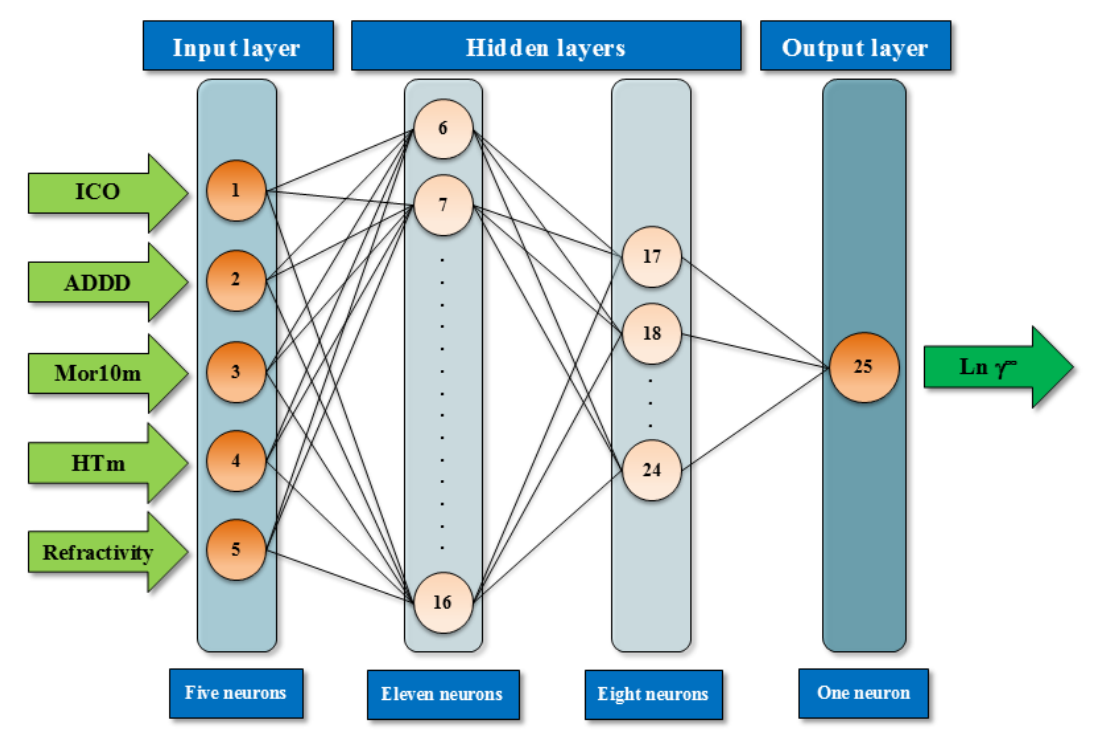

Fig. 9. Topology of Artificial Neural Network (5-11-8-1). 
As quote above, the specialization of input neurons is marked by the value of the weights of each neuron with the first intermediate layer. The absolute-value sum of the weights of each neuron contributes to determine the importance of the input variable to predict the wanted value. Table 6 shows the weight values of each Neural Network's neuron. Here, we can also see the importance of each input variable to predict the output variable. According to this, there are two major variables, i) HTm that corresponds with the $\mathrm{H}$ total index/weighted by atomic masses with an importance of $26.79 \%$ and ii) ADDD that corresponds with the average distance/distance degree with $23.84 \%$. The least important input variable is Mor $10 \mathrm{~m}$ that denotes the 3D-Morse- signal 10/weighted by atomic masses $(14.93 \%)$

Table. 6. Weights between input layer and first intermediate layer (light grey), weights between first and second intermediate layers (light yellow), weights between second intermediate layer and output layer (light red), importance of variables in percentage (light blue), and finally, biases (light green) for the best implemented Neural Network (5-11-8-1).

\begin{tabular}{|c|c|c|c|c|c|c|c|c|c|c|c|}
\hline ICO & ADDD & Mor10m & HTm & $\begin{array}{c}\text { Refractivit } \\
y\end{array}$ & & \multicolumn{2}{|c|}{ Variable } & \multicolumn{2}{|c|}{$\begin{array}{c}\text { Importance } \\
(\%)\end{array}$} & & bias \\
\hline 0.98 & -1.65 & -3.53 & 2.85 & 3.40 & & HTm & & & & & -3.56 \\
\hline-2.72 & 4.16 & 1.36 & 0.96 & 0.05 & & ADDD & & & & & -1.84 \\
\hline-0.74 & 2.08 & -0.95 & 0.50 & 1.51 & & ICO & & & & & -4.57 \\
\hline 1.76 & 6.26 & 2.92 & -11.78 & 2.19 & & Refrac & ivity & & & & -0.29 \\
\hline-0.49 & 1.04 & -0.53 & 0.70 & -0.08 & & Mor10 & & & & & -3.19 \\
\hline-3.91 & -0.48 & 0.58 & 5.05 & 2.20 & & & & & & & -1.96 \\
\hline-0.48 & 2.86 & 1.61 & 2.31 & -1.40 & & & & & & & -4.32 \\
\hline-1.35 & 2.74 & -1.13 & 1.02 & 2.83 & & & & & & & -5.43 \\
\hline-6.36 & 1.17 & 2.08 & 2.20 & 0.83 & & & & & & & 3.15 \\
\hline-1.05 & 2.54 & -0.81 & 0.58 & 2.27 & & & & & & & -5.18 \\
\hline-0.51 & 0.73 & -0.61 & 0.95 & 0.02 & & & & & & & -3.06 \\
\hline-0.93 & -0.84 & 0.12 & -2.31 & -0.30 & -1.54 & -0.89 & 0.31 & -0.86 & 0.35 & -0.39 & 0.10 \\
\hline-3.30 & 2.27 & 0.24 & -1.65 & -0.22 & -3.89 & 2.32 & 0.28 & -1.90 & 0.47 & -0.37 & 0.58 \\
\hline 1.87 & -1.25 & 0.67 & -1.42 & -0.53 & 0.68 & -3.87 & 1.66 & 2.25 & 1.27 & -0.56 & 0.93 \\
\hline-1.23 & -0.53 & 0.10 & -2.16 & -0.29 & -1.70 & -0.55 & 0.25 & -0.99 & 0.32 & -0.39 & 8.76 \\
\hline 0.04 & -0.80 & -2.46 & -0.39 & -1.09 & 3.85 & 0.52 & -3.62 & -3.47 & -3.02 & -0.99 & 1.83 \\
\hline-0.78 & -1.50 & -0.03 & -2.70 & -0.30 & -1.51 & -0.99 & 0.09 & -1.22 & 0.17 & -0.39 & 0.60 \\
\hline-1.81 & 0.19 & 0.10 & -1.94 & -0.28 & -2.04 & 0.19 & 0.20 & -1.23 & 0.31 & -0.39 & 1.82 \\
\hline-0.76 & -1.51 & -0.02 & -2.70 & -0.30 & -1.50 & -1.02 & 0.10 & -1.20 & 0.18 & -0.39 & 8.33 \\
\hline-3.16 & -3.37 & -2.07 & -3.02 & -4.38 & -3.59 & -2.84 & -3.59 & & & & \\
\hline
\end{tabular}

\section{Conclusion}

An Artificial Neural Network with a low margin of error in the prediction of activity coefficients at infinite dilution has been implemented. The best Artificial Neural Network has a 5-11-8-1 topology, it has been trained with 75 cases and 30 cases validated for 64,000 cycles. This ANN presents a $\mathrm{R}^{2}$ of 0.990 in the training phase, a RMSE of 0.277 and an APD of $1.85 \%$. For the validation phase, it presents a $\mathrm{R}^{2}$ of 0.986 , a RMSE of 0.291 and an Average Percentage Deviation of $1.88 \%$.

In terms of Root Mean Square Error and Average Percentage Deviation, the implemented Neural Network model improves the prediction model based in ACO-MLR ${ }^{1}$, both during the 
training phase as in the validation phase. These improvements, in terms of RMSE, are $30.70 \%$ and $24.60 \%$ for the training and validation phase, respectively.

To sum up, the back propagation Neural Network model has a high predictive power, and it does not show outliers. In fact, the results obtained by the Neural Network advise the use of Artificial Neural Networks to estimate the activity coefficient at infinite dilution using the ACO method, which determine the optimal descriptors of hydrocarbons in water that are significant to predict them.

New insights in order to save resources, materials, money and time needed to develop the experimental method to determine the activity coefficient at infinite dilution would be saved with the use of the present prediction model.

\section{Acknowledgements}

G. Astray thanks Xunta de Galicia, Consellería de Cultura, Educación e Ordenación Universitaria, for the Postdoctoral Grant (Plan I2C), P.P.0000 421S 14008 and CIA Project, P.P. K224 131H 6450211 for financial support to develop this research. J. Morales thanks the University of Vigo for a research-training Grant (P.P. 0022 122I 641.03).

\section{References}

1- M. Atabati, K. Zarei and A. Borhani, Fluid Phase Equilibr., 2010, 293, 219-224.

2- Y. L. Cao and H. Y. Li, Chinese Chemi. Lett., 2003, 14, 987-990.

3- K. Zarei and M. Atabati, J. Solution Chem., 2013, 42, 516-525.

4- J. Xu, L. Wang, L. Wang, H. Zhang and W. Xu, J. Solution Chem., 2011, 40, 118-130.

5- R. Martínez, M. T. Sanz, S. Beltrán and E. Corcuera, J. Chem. Eng. Data, 2012, 57, 1480-1485.

6- S. Nebig, V. Liebert and J. Gmehling, Fluid Phase Equilibr, 2009, 277, 61-67.

7- V. Brandani, Ind. Eng. Chem., 1974, 13, 154-156.

8- S. Kato, Y. Hata and T. Hibino, Solvent Extr. Res. Dev., 2009, 16, 121-125.

9- K. Kojima, S. Zhang and T. Hiaki, Fluid Phase Equilib., 1997, 131, 145-179

10- J. Gmehling, Activity Coefficients at Infinite Dilution-Measurement and Application, 2012, http://www.gmehling.chemie.uni-oldenburg.de/9721.html.

11- T. M. Letcher, B. Soko, P. Reddy and N. Deenadayalu, J. Chem. Eng. Data, 2003, 48, 1587-1590.

12- D. Gruber, M. Krummen and J. Gmehling, Chem. Eng. Technol., 1999, 22, 827-831.

13- C. Zhao Jr. and J. Li Sr., ACS National Meeting Book of Abstracts, 2005, 230.

14- K. Tochigi and J. Gmehling, J. Chem. Eng. Japan, 2011, 44, 304-306.

15- K. Fukuchi, T. Watanabe, S. Yonezawa and Y. Arai, J. Chem. Eng. Japan, 1998, 31, 667-669.

16- B. G. Choi, J. S. Choi, K. Tochigi and K. Kojima, J. Chem. Eng. Japan, 1996, 29, 217-222.

17- J. C. Bastos, M. E. Soares and A. G. Medina, Ind. Eng. Chem. Res., 1988, 27, 1269-1277.

18- J. Wang, W. Sun, C. Li and Z. Wang, Fluid Phase Equilibr., 2008, 264, 235-241.

19- F. Giralt, G. Espinosa, A. Arenas, J. Ferre-Gine, L. Amat, X. Gironés, R. Carbó-Dorca and Y. Cohen, AIChE J., 2004, 50, 1315-1343.

20- J. He and C. Zhong, Fluid Phase Equilibr., 2003, 205, 303-316.

21- M. Ge, C. Li and J. Ma, Electrochemistry, 2009, 77, 745-747.

22- S. Ajmani, S. C. Rogers, M. H. Barley, A. N. Burgess and D. J. Livingstone, QSAR Comb. Sci., 2008, 27, 1346-1361.

23- H. Xu and J. Min, Jiegou Huaxue, 2008, 27, 491-497. 
24- F. Nami and F. Deyhimi, J. Chem Thermodyn., 2011, 43, 22-27.

25- M. Atabati, K. Zarei and M. Mohsennia, Anal Chim Acta, 2010, 663, 7-10.

26- M. Atabati and F. Khandani, Chinese Chemi. Lett., 2012, 23, 1209-1212.

27- G. Astray, M. A. Iglesias-Otero, O. A. Moldes and J. C. Mejuto, Tenside Surfact. Det, 2013, 50, 118-124.

28- G. Astray, J. F. Gálvez, J. C. Mejuto, O. A. Moldes and I. Montoya, J. Comp. Chem., 2013, 34, 355-359.

29- E. Okumura, I. Kawashita and T. Ishida, J. Digital Imaging, 2011, 24, 1126-1132.

30- W. Penny and D. Frost, Med. Decis. Making, 1996, 16, 386-398.

31- G. Astray, P. V. Caderno, J. A. Ferreiro-Lage, J. F. Galvez and J. C. Mejuto, J. Chem. Eng. Data, 2010, 55, 3542-3547.

32- Y. Tian, J. Zhang and J. Morris, Chem. Eng. Process, 2002, 41, 531-538.

33- J. Gasteiger and J. Zupan, Angew. Chem. Int. Edit., 1993, 32, 503-527.

34- M. W. Gardner and S. R. Dorling, Atmos. Environ., 1998, 32, 2627-2636.

35- G. Astray, F. J. Rodríguez-Rajo, J. A. Ferreiro-Lage, M. Fernández-González, V. Jato and J. C. Mejuto, J. Environ. Monitor., 2010, 12, 2145-2152.

36- J. S. Torrecilla, L. Otero and P. D. Sanz, J. Food Eng., 2004, 62, 89-95.

37- G. Astray, J. X. Castillo, J. A. Ferreiro-Lage, J. F. Galvez and J. C. Mejuto, Ciencia y Tecnologia Alimentaria, 2010, 8, 79-86. 